\title{
Prospects of 5G Communications
}

\author{
Mastaneh Mokayef, ${ }^{1}$ Yasser Zahedi ${ }^{2}$ \\ ${ }^{1}$ Faculty of Engineering, Technology and Built Environment UCSI university, 56000, Kuala Lumpur, Malaysia \\ ${ }^{2}$ Wireless Communication Center (WCC) University Technology Malaysia, 81310, Johor, Malaysia \\ ${ }^{1}$ mastaneh@ucsiuniversity .edu .my \\ ${ }^{2}$ yasserzahediegmail.co
}

\begin{abstract}
The next generation of wireless communication is going to meet human demands beyond today's trend. This study sets the frame on the future of wireless communication that requires real time responses which pushes this technology towards lower latency and conflicting required band for whole system. Moreover, the challenges for this hot topic are highlighted. So this study had shed light on the important studies that had been done to get to the bottom of these issues.
\end{abstract}

Keywords-5G, millimeter-wave, channel model, spectrum allocation.

\section{INTRODUCTION}

From the long time scale, the first huge success of the wireless communication is the combination of video and mobile technology with the telephony technology that is the achievement of Global System for Mobile Communications (GSM) network. The GSM network simply moved the fixed voice service into the mobile network that was a huge success [1]. Later, in 1998 the concern was to use the combination of mobile network with internet service. Hence the basic $3 \mathrm{G}$ has been introduced by Code Division Multiple Access (CDMA) and the 3rd Generation Partnership Project (3GPP). The 3GPP covered cellular telecommunications network technologies, including radio access, the core transport network, and service capabilities, moreover, considering the security and quality of service (QoS) has led to complete system specifications. The specifications provide hooks for non-radio access to the core network, and also cooperation of new technology with Wi-Fi networks. The main concern of those days was to make the swift network with the speed of 10 times of the GSM which practically led to our accessibility to the internet. Then the High Speed Packet Access (HSPA) came up which increased the speed of internet access up to ten times. So in the process of changing technology and aiming the internet access in addition to the voice service, the Long Term Evolution (LTE) came up and is accessible nowadays.

Going to the next generation of wireless communications (5G), not only the internet access is going to be offered to the user, it is much more diverse service which is connecting everything in the world. This definition will show how vast the $5 \mathrm{G}$ systems are. Therefore, $5 \mathrm{G}$ enables the connection of everyone and everything in anytime and anywhere known as Internet of Things (IOT).

Main benefits of adopting 5G concept are basically the higher capacity, higher data rate, ultra-low latency, high reliability of applications and more energy efficiency. In order to support high required data traffic in future communication systems, $5 \mathrm{G}$ is supposed to provide 1000 times more capacity in comparison to $4 \mathrm{G}$ in the hierarchy network in parallel with 100 times faster speed and 100 times more connectivity to the users. The most important issue regarding $5 \mathrm{G}$ is the ultra-low latency which would be beyond human perception. Two way gaming, cloud based technologies, tactile internet, and virtual realities require much less roundtrip latencies compared to $4 \mathrm{G}$. Consequently, 5G latencies are intended to be around $1 \mathrm{~ms}$, which is so small amount in comparison to $15 \mathrm{~ms}$ for $4 \mathrm{G}$ case. For future wireless systems, the design of new applications is pictured based on machine-to-machine (M2M) communication with real-time embarrassment to improve the quality of life. Enhanced mobile cloud services, real-time traffic control optimization, emergency and disaster response, smart grid, e-health or efficient industrial communications are also examples of where low latency and high reliability in the coming $5 \mathrm{G}$ technology appears. The last but not the least is energy which will change the architecture of the network for deployment. Energy efficiency is one of the requirements for $5 \mathrm{G}$ systems. Besides the Spectrum Efficiency (SE), the Energy Efficiency (EE) has been also introduced as a critical issue for the next generation of wireless communications.

Fundamentally $5 \mathrm{G}$ can be defined as the continued evolution of mobile broadband service based on the cellular network which requires 100 times more throughput. The technical challenge for this proposed technology is the higher spectrum efficiency. $5 \mathrm{G}$ is a cellular-based IOT that has a large coverage. Older networks are also in the center of attention to be virtualized and be able to arbitrary change topologies due to the service.

Getting to more detail, $5 \mathrm{G}$ is more than just a high speed communication. Hence the links of the network as well as 
latency are all together making the 5G concept. $3 \mathrm{G}$ and $4 \mathrm{G}$ networks are designed for only two parameters which are average spectrum efficiency and average cell spectrum efficiency[2]. Hence, in order to design 5G, the network has to be very dynamically virtualized and being abstracted by the applications. That would require a definable physical layer.

At this very early stage, the spectrum shortage is one of the most important points that must be taken into account. The other challenge regarding this new technology is its high efficiency. Accordingly, seeking disruptive spectral efficiency for a revolutionary clean slate design is of the biggest concern. Proposing a high gain for $5 \mathrm{G}$, the frequency spectrum below $6 \mathrm{GHz}$ is still very critical to lunch $5 \mathrm{G}$ and adding the internet of the things. So the spectrum efficiency is the key challenge in lunching the $5 \mathrm{G}$.

The potential of $5 \mathrm{G}$ can be easily illustrated by thinking of the IOT. Massive connections of things require a huge number of sensors to make it possible, considering the connection of everything in addition to the already existing human gadget connections. The other critical issue in controlling the IOT for the purpose of optimizing the connectivity of different things is the zero latency for $5 \mathrm{G}$.

The remainder of the paper is arranged as follows: Section II describes the required physical layer, while section III briefly introduces the Multiple-input Multiple -Output (MIMO) technology and proposed channel models for 5G. In section $\mathrm{IV}$, the spectrum regulations for $5 \mathrm{G}$ are presented. Finally, the paper is concluded in Section.

\section{PHYSICAL LAYER}

Studies [3-8] have been conducted in order to propose a proper physical layer for the practical operation of $5 \mathrm{G}$. The common sense in all these studies is departing from LTE-OFDM due to the difference between $4 \mathrm{G}$ and $5 \mathrm{G}$ fundamental constructions. In case of $4 \mathrm{G}$, the construction makes the need of uniform orthogonality and one computing platform for the file transfer protocol (FTP) with strict synchronization of different kinds of services that limits lots of applications in $4 \mathrm{G}$.

Therefore, the proposed framework for $5 \mathrm{G}$ is based on departing from the two layers of true orthogonal and synchronized layer into the non-orthogonal and synchronous-free structure to enable the IOT. The first layer uses Flash Orthogonal Frequency Division Multiplexing (F-OFDM) which applies filtering on the resource block to decouple the inter-block that is existed in $4 \mathrm{G}$ structure. Some blocks can use ultra-narrowband while some others are using very high internet speed, some with very small cyclic preface and utilized for indoor but they are all in the harmonized design, if we have inter-block orthogonality.

An extra dimension more than the orthogonality for the case of $5 \mathrm{G}$ is required. This dimension is where the spectrum efficiency will come out with the first basic step. In this case a very low latency and very large number of link connectivity would be provided.

\section{ARCHITECTURE}

The ever increasing demand of the wireless communication led to the new efficient architecture for the upcoming $5 \mathrm{G}$ to address the so called "Exabyte data flood" which is two level above terabytes [9].

The intervention of the future system would be significantly improved by changing the deployment of the system architecture to allow the system to be connected to several local and remote control centers in rapid and consistent manner. Hence a brief review on the structure of Multi-Input Multi-Output (MIMO) technology as one of the key factors of $5 \mathrm{G}$ issues to support more data rate of about 1000 times $4 \mathrm{G}$, and the channel model is illustrated in this section [1-5].

One of the biggest challenges regarding $5 \mathrm{G}$ is the realization of the massive MIMO due to the $5 \mathrm{G}$ structure. Due to the small cell size in $5 \mathrm{G}$, the need for massive MIMO (instead of conventional MIMO) for these congested small cells will increase. In massive MIMO systems, the antenna arrays can have a few hundred antennas and able to serve tens of terminals in the same time-frequency resource [10]. Consequently, the advantages of using conventional MIMO will be conserved and observed on a much greater scale. One of the benefits of Massive MIMO is the effects of Gaussian noise and uncorrelated intra-cell and inter-cell interference are eliminated in Massive MIMO. In a massive MIMO system equipped with many low power antennas, better performance regarding power saving, spectrum efficiency and link reliability can be achieved compared to conventional MIMO. Massive MIMO can be used as an alternative to cell size shrinking.

The capacity of Massive MIMO system is increased as the number of the equipped antennas increases. However, some technical issues should be taken in this regard such as; pilot and low power transmission design from the practical perspective along with channel estimation, power allocation strategies and capacity bounds from the theoretical perspective [11]. Other MIMO limiting factors can be narrated to channel reciprocity, pilot contamination, radio propagation and orthogonality of channel responses [10]. 
Regarding the channel modeling and characterization targeting the 5G deployment, wide field measurements are required to establish the proper channel modeling based on the impact of antenna correlation and couplings $[12,13]$.The current channel model proposed for the future operation of $5 \mathrm{G}$ is a $3 \mathrm{D}$ channel modeling[14] . According to [15], the WINER I project is already focused on the channel model to cover a proper propagation scenario for all indoor, urban micro and macro cells, suburban and rural macro cells and the stationary feeder links, scenarios. Moreover, the WINER II project is focusing on prediction of large scale path loss statistics. In addition, the other considerable channel model is the one utilized for the International Mobile TelecommunicationAdvanced (IMT-A).

Most recently, some channel measurements have been conducted assuming the millimeter wave (mm-wave) spectrum for the operation of 5G. Subsequently, studies [16-22] have performed different propagation measurements at $28 \mathrm{GHz}$ and $38 \mathrm{GHz}$. Regarding these studies, the micro cell radius of 200 meters with the single 10-degree beam-width antenna provided the cell coverage of $80 \%$ for combined four coherent beams. The beam combination procedure have been performed using float intercept model in [23] and the close-in free space reference path loss model have been conducted in [24].

According to the obtained results the beam combining improves the received signal quality and link coverage. More specified result regarding beam combination can be found in [24] in which the combination of bi-beam, tri-beam and quad-beam and their effect on path loss and shadow has been investigated. Consequently, the revealed results proved the effect of combining four beams will led to reduction in transmitter-receiver separation distance of up to 100 meters. Table 1 illustrates the comparison between current 2 to 4 generations and the 5th Generation.

Table 1. Comparison between current generations and 5G.

\begin{tabular}{|l|c|c|}
\hline & Current generation & $5 \mathrm{G}$ \\
\hline subscription & 7.085 million & 7.02 billion \\
\hline IOT units & 8 million & 26 billion \\
\hline $\begin{array}{l}\text { Internet connected } \\
\text { devices per person }\end{array}$ & 1.8 & 6.8 \\
\hline
\end{tabular}

The other issue concerned in $5 \mathrm{G}$ structure is to propose this new technology to serve public safety users such as military forces relying on channel condition sub-grouping policies. In this scenario, the quality of channel is the key element on priority decision i.e., the user with poor channel condition has priority while maximizing user's throughput.
The standalone ground and areal platforms are main components of this structure in order to have a rapid deployment in disaster scenarios where the physical access is blocked.

The last but not the least is the cell structure that must be designed in such a way to be able to use wireless backhaul for transmitting data to other cells by means of fiber optic links. At this juncture, the cell size is in the center of attention. The cell must be designed with a significant size to provide the required frequency reuse while minimizing the number of base stations that leads to the cost efficiency of cell $[19,25]$. Therefore, the cell coverage extension for a specified transmitter height is investigated in [26] . Consequently to have a new generation of wireless communication beyond our today's smart phones, all the above mentioned challenges must be taken into account while proposing the new architecture for $5 \mathrm{G}$.

\section{SPECTRUM ALLOCATION AND CHALLENGES}

Considering the fast growth of demand for mobile broadband subscriptions of 7.6 billion subscribers by end of the year 2019 [9], the spectrum allocation and management became the center of attention for researchers around the world. When we first hear about the spectrum, we most probably look for the licensed ones but if we think more realistic, will find out that there is no need to limit ourselves to the licensed spectrum while there are unlicensed spectrum already existed. The unlicensed spectrum is usually used for $\mathrm{Wi}-\mathrm{Fi}$ and local area networks.in this case we use the licensed spectrum to control the operations of unlicensed ones.

Increasing the bandwidth is in a center of attention in the future emerged technology as it is supposed to provide the 1000 times higher data rate than today's one. Hence the easiest way to achieve this goal is to move the operating spectrum to the mm-wave spectrum with the wavelength of 10 millimeters and frequency of $30 \mathrm{GHz}$ and above. The millimeter wave bands also promise the use of high number of unlicensed spectrum around $28 \mathrm{GHz}$ and $38 \mathrm{GHz}$. The other reason to consider this frequency band for the proposed $5 \mathrm{G}$ is its negligible and resistance to the rain attenuation [17, 21] which is an essential issue in the tropical countries like Malaysia.

Based on studies in $[7,10]$ the $28 \mathrm{GHz}, 38 \mathrm{GHz}, 60 \mathrm{GHz}$, $70 \mathrm{GHz}$ and $90 \mathrm{GHz}$ can potentially be proper candidates for next wireless generation. Among these bands, the $28 \mathrm{GHz}$, 71-76 GHz, and 81-86 GHz bands are excellent candidates for $5 \mathrm{G}$ deployment as they operate under Federal Communication and Commission (FCC) Part 101 rules and are allocated for fixed microwave services, Fixed Satellite services (FSS) and point to point microwave operations. In addition, 
considering the small cells of about 200 meters radii, the rain attenuation is negligible in these frequency bands. The reduction in cell size will cause the spatial load reduction and spectral aggregation at the same time. This it-self will increase the spectral efficiency by coexisting the heterogeneous networks (Het Net) [9]. Due to the reduction of cell size, the electromagnetic spectrum scarcity would be increased hence the mitigation techniques such as Dynamic Spectrum Access (DSA) would be required to overcome this problem. Accordingly, the cognitive radio would be more engaged to enable the DSA.

The other point to consider about the mentioned bands is their potential to use the mobile allocation in ITU-R Radio Regulations. 5G standardization status will be shaped based on the released results of several projects that have been conducted all over the world. Up to date, the European Union has already released their regulations and requirements based on their successful METIS project while, International Telecommunications Union (ITU) working group is focused on the obtained results from different industries and referred 5th Generation of wireless to "IMT-2020". However, the European Telecommunications Standard Institute (ETSI) concluded that the evolution of LTE may not be sufficient for the $5 \mathrm{G}$ necessities.

\section{CONCLUSION}

Hyper dense small cells will bring our dreams of having smart life, 1000 times closer to the reality. The feasibility of different spectrum including millimeter wave band has to be proposed by national regulators for mobile applications and the final decision would be made by ITU world radio communication (WRC), hence, all market-based regulations, network planning, resource allocations, licensed and unlicensed spectrum regulations and the economic situations must be taken into account in order to establish the proper regulations for the next generation of wireless communications. The exciting time in wireless industry is began thinking of millimeter wave utilization rather than a fantasy. Hopefully, the millimeter wave bands provide the results as what have been indicated in the outage studies in all over the world. As higher frequencies are used for the $5 \mathrm{G}$ systems, some techniques such as the massive MIMO can be used in order to provide range extension from the base station antenna to mobile terminals antennas in addition to the capability of increasing the data rate. Finally, the main aspects of the future cellular communication are based on the higher data rate and the more energy efficiency.

\section{REFERENCES}

[1] T. V. N. Rao, S. A. Aasha and S. Tirumalaraju. "5G technologies-an anecdote of network service for the future," Journal of Global Research in Computer Science, vol. 2, no. 7, pp: 164-170, 2011.

[2] C.-X. Wang, F. Haider, X. Gao, X-H. You, Y. Yang, D. Yuan, H. Aggoune, H. Haas, S. Fletcher and E. Hepsaydir. "Cellular architecture and key technologies for $5 \mathrm{G}$ wireless communication networks," IEEE Communications Magazine, vol. 52, no. 2, pp: 122-130, 2014.

[3] T.Janevski. "5G mobile phone concept," In Proceedings of the $6^{\text {th }}$ IEEE Conference on Consumer Communications and Networking, 2009, pp: 823-824.

[4] P. Mogensen, K. Pajukoski, E. Tiirola, E. Lähetkangas, J. Vihriälä, S. Vesterinen, M. Laitila, G. Berardinelli, G. W. O. Da Costa, L. G. U. Garcia, F. M. L. Tavares and A. F. Cattoni. "5G small cell optimized radio design," In Proceedings of IEEE Globecom Workshops, 2013.

[5] S. Chen and J. Zhao. "The requirements, challenges, and technologies for $5 \mathrm{G}$ of terrestrial mobile telecommunication," IEEE Communications Magazine, vol. 52, no. 5, pp: 36-43, 2014.

[6] G. Wunder, M. Kasparick, S. T. Brink, F. Schaich, T. Wild, Y. Chen, I, Gaspar, N. Michailow, G. Fettweis, D. Ktenas, N. Cassiau, M. Dryjanski, K. Sorokosz, S. Pietrzyk and B. Eged. "System-level interfaces and performance evaluation methodology for $5 \mathrm{G}$ physical layer based on non-orthogonal waveforms," In Proceedings of 2013 Asilomar Conference on Signals, Systems and Computers, 2013.

[7] G. Wunder, P. Jung, M. Kasparick, T. Wild, F. Schaich, Y. Chen, S. T. Brink, I. Gaspar, N. Michailow, A. Festag, L. Mendes, N. Cassiau, D. Ktenas, M. Dryjanski, S. Pietrzyk, B. Eged, P. Vago and F. Wiedmann. "5GNOW: non-orthogonal, asynchronous waveforms for future mobile applications," IEEE Communications Magazine, vol. 52, no. 2, pp: 97-105, 2014.

[8] B. Bangerter, S. Talwar, R. Arefi and K. Stewart. "Networks and devices for the 5G era," IEEE Communications Magazine, vol. 52, no. 2, pp: 90-96, 2014.

[9] S. A. R. Zaidi, A. Afzal, M. Hafeez, M. Ghogho, D. C. Mclernon and A. Swami. "Solar energy empowered 5G cognitive metro-cellular networks," IEEE Communications Magazine, vol. 53, no. 7, pp: 70-77, 2015.

[10] E. G. Larsson, O. Edfors, F. Tufvesson and T. L. Marzetta. "Massive MIMO for next generation wireless systems," IEEE Communications Magazine, vol. 52, no. 2, pp: 186-195, 2014.

[11] X. Su, J. Zeng, L-P. Rong and Y-J. Kuang. "Investigation on Key Technologies in Large-Scale MIMO," Journal of Computer Science and Technology, vol. 28, no. 3, pp: 412-419, 2013. 
[12] Y-H. Nam, B. L. Ng, K. Sayana, Y. Li, J. Zhang, Y. Kim and J. Lee. "Full-dimension MIMO (FD-MIMO) for next generation cellular technology," IEEE Communications Magazine, vol. 51, no. 6, pp. 172-179, 2013.

[13] S. Wu, C-X. Wang, El-H. M. Aggoune, M. M. Alwakeel and Y.He. "A non-stationary 3-D wideband twin-cluster model for 5G massive mimo channels," IEEE Journal on Selected Areas in Communications, vol. 32, no. 6, pp: 1207-1218, 2014.

[14] Y-H. Nam, Y. Li, J. C. Zhang. "3D channel models for elevation beamforming and FD-MIMO in LTE-A and 5G," In Proceedings of $48^{\text {th }}$ Asilomar Conference on Signals, Systems and Computers, 2014.

[15] K. Gomez, L. Goratti, F. Granelli and T. Rasheed. "A comparative study of scheduling disciplines in $5 \mathrm{G}$ systems for emergency communications," In $1^{\text {st }}$ International Conference on $5 G$ for Ubiquitous Connectivity (5GU), 2014.

[16] G. R. MacCartney, J. Zhang, S. Nie and T. S. Rappaport. "Path loss models for 5G millimeter wave propagation channels in urban microcells," In Proceedings of Global Communications Conference (GLOBECOM), 2013.

[17] Y. Azar, G. N. Wong, K. Wang, R. Mayzus, J. K. Schulz, H. Zhao, F. Gutierrez, D-D. Hwang and T. S. Rappaport. " $28 \mathrm{GHz}$ propagation measurements for outdoor cellular communications using steerable beam antennas in New York City," In Proceedings of IEEE International Conference on Communications (ICC), 2013.

[18] T. S. Rappaport, S. Sun, R. Mayzus, H. Zhao, Y, Azar, K. Wang, G. N. Wong, J. K. Schulz, K. Samimi and F. Gutierrez. "Millimeter wave mobile communications for 5G cellular: It will work!," IEEE Access, vol. 1, pp: 335-349, 2013.

[19] T. S. Rappaport, F. Gutierrez, E. Ben-Dor, J. N. Murdock, Y. Qiao and J. I. Tamir. "Broadband millimeter-wave propagation measurements and models using adaptive-beam antennas for outdoor urban cellular communications," IEEE Transactions on Antennas and Propagation, vol. 61, no. 4, pp: 1850-1859, 2013.

[20] E. Ben-Dor, T. S. Rappaport, Y. Qiao, and S. J. Lauffenburger. "Millimeter-wave $60 \mathrm{GHz}$ outdoor and vehicle AOA propagation measurements using a broadband channel sounder," In Proceedings of Global Telecommunications Conference (GLOBECOM 2011), 2011.

[21] M. Samimi, k. Wang, Y. Azar, G. N. Wong, R. Mayzus, H. Zhao, J. K. Schulz, S. Sun, F. Gutierrez and T. S. Rappaport. " $28 \mathrm{GHz}$ angle of arrival and angle of departure analysis for outdoor cellular communications using steerable beam antennas in New York City," In Proceeding of IEEE 77th Vehicular Technology Conference (VTC Spring), 2013.

[22] S. Piersanti, L. A. Annoni and D. Cassioli. "Millimeter waves channel measurements and path loss models," In Proceedings of 2012 IEEE International Conference on Communications (ICC), 2012.

[23] S. Sun, and T. S. Rappaport. "Multi-beam antenna combining for $28 \mathrm{GHz}$ cellular link improvement in urban environments," In Proceedings of 2013 IEEE Global Communications Conference (GLOBECOM), 2013.

[24] S. Sun, G. R. MacCartney, M. K. Samimi, S. Nie and T. S. Rappaport. "Millimeter wave multi-beam antenna combining for $5 \mathrm{G}$ cellular link improvement in New York City," In Proceedings of 2014 IEEE International Conference on Communications (ICC), 2014.

[25] H. R. Anderson, Fixed broadband wireless system design. John Wiley \& Sons, 2003.

[26] J. N. Murdock, E. Ben-Dor, Y. Qiao, J. I. Tamir and T.S. Rappaport. "A $38 \mathrm{GHz}$ cellular outage study for an urban outdoor campus environment," In Proceedings of 2012 IEEE Wireless Communications and Networking Conference (WCNC), 2012.

(C) Author(s) 2016. CC Attribution 4.0 License. (http://creativecommons.org/licenses/by-nc/4.0/)

This article is licensed under the terms of the Creative Commons Attribution Non-Commercial License which permits unrestricted, non-commercial use, distribution and reproduction in any medium, provided the work is properly cited. 